\title{
Histologic changes of nonbiodegradable and biodegradable biomaterials used to repair right ventricular heart defects in rats
}

Tsukasa Ozawa, MD, PhD, Donald A. G. Mickle, MD, MSc, Richard D. Weisel, MD, Nobuya Koyama, MD, PhD, Harvey Wong, BSc, Sumiko Ozawa, MD, and Ren-Ke Li, MD, PhD

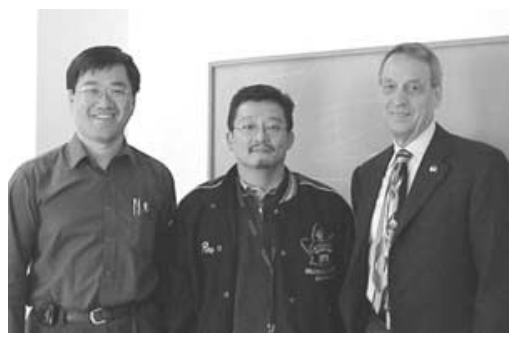

Li, Ozawa, and Weisel (left to right)

Objectives: Nonbiodegradable synthetic materials have been widely used to repair cardiac defects. Material-related failures, however, such as lack of growth, thrombosis, and infection, do occur. Because a biodegradable scaffold can be replaced by the patient's own cells and will be treated as a foreign body for a limited period, we compared four biodegradable materials (gelatin, polyglycolic acid (PGA), and copolymer made of $\epsilon$-caprolactone and L-lactic acid reinforced with a poly-L-lactide knitted [KN-PCLA] or woven fabric [WV-PCLA]) with a nonbiodegradable polytetrafluoroethylene (PTFE) material. An animal heart model was tested that simulates the in vivo clinical condition to which a synthetic material would be used.

Methods: The five patches were used to repair transmural defects surgically created in the right ventricular outflow tracts of adult rat hearts ( $\mathrm{n}=5$, each patch group). The PTFE patch group served as a control group. At 8 weeks after implantation, the

From the Department of Surgery, Division of Cardiovascular Surgery, Toronto General Research Institute, Toronto General Hospital, and University of Toronto, Toronto, Ontario, Canada.

R.K.L. is supported by research grants funded by Heart \& Stroke Foundation of Ontario (NA\#4603 and NA\#4829) and Canadian Institute of Health Research (MOP14795). R.K.L. is a Career Investigator of the Heart and Stroke Foundation of Canada.

Received for publication Feb 4, 2002; revisions requested May 15, 2002; revisions received June 10, 2002; accepted for publication June 14, 2002.

Address for reprints: Ren-Ke Li, MD, PhD, Toronto General Hospital, CCRW 1-815, 200 Elizabeth St, Toronto, Ontario M5G 2C4, Canada (E-mail: RenKeLi@uhnres. utoronto.ca).

J Thorac Cardiovasc Surg 2002;124: 1157-64

Copyright (C) 2002 by The American Association for Thoracic Surgery

0022-5223/2002 \$35.00+0 12/1/127449

doi:10.1067/mtc.2002.127449 biomaterials were excised. Patch size, patch thickness, infiltrated cell number, extracellular matrix composition, and patch degradation were evaluated.

Results: The PTFE patch itself did not change in size except for increasing in thickness because of fibroblast and collagen coverage of both its surfaces. Host cells did not migrate into the PTFE biomaterial. In contrast, cells migrated into the biodegrading gelatin, PGA, and KN-PCLA and WV-PCLA scaffolds. Cellular ingrowth per unit patch area was highest in the KN-PCLA patch. The KN-PCLA patch increased modestly in size and thinness. The WV-PCNA patch did not change in size or thickness. Fibroblasts and collagen were the dominant cellular infiltrate and extracellular matrix formed in the biodegrading scaffolds. The in vivo rates of biomaterial degradation, thinning, and expansion were material specific. All the subendocardial patch surfaces were covered with endothelial cells. No thrombi were seen.

Conclusions: The unique, spongy matrix structure of the PCLA patch favored cell colonization relative to the other patches. The strong, durable outer poly-L-lactide fabric layers in these patches offered physical, biocompatible, and bioresorbable advantages relative to the other biodegradable materials studied. Host cells migrated into all the biomaterials. The cells secreted matrix and formed tissue, which was endothelialized on the endocardial surface. The biomaterial degradation rates and the tissue formation rates were material related. The PCLA grafts hold promise to become a suitable patch for surgical repair. 


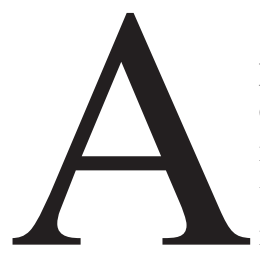

conduit from the right ventricle to the pulmonary artery has made possible the correction of numerous complex congenital cardiac anomalies that previously were uncorrectable. ${ }^{1}$ A transannular patch is also mandatory for children with tetralogy of Fallot, double-outlet right ventricle with pulmonary stenosis, or the similar anomalies in the right ventricular outflow tract (RVOT). Currently, the conduits or patches are made of Dacron polyester fabric, polytetrafluoroethylene (PTFE), glutaraldehyde-treated bovine pericardium, or antibiotic-preserved or cryopreserved homografts. ${ }^{2}$ The longterm results have been compromised by material-related failures. ${ }^{3}$ The materials are not viable, do not grow, and do not provide pulsatile flow. Lack of growth of the surgical biomaterial can necessitate reoperations to replace the patch or conduit because of the child's growth. ${ }^{4}$ Synthetic material is treated by the patient's immune system as a foreign body and may become thrombogenic and infected.

Since the biodegradable scaffold needs to be capable of resisting damage from the contracting myocardium and be bioresorbable at a rate compatible with the repair process, we studied the in vivo characteristics of acellular scaffolds used to repair a surgically created myocardial defect in the right ventricle of the adult rat. Nonbiodegradable PTFE and biodegradable gelatin sponge, biodegradable nonwoven polyglycolic acid (PGA) mesh, and biodegradable poly-Llactide knitted or woven fabric with a $50 \%$-caprolactone and 50\% L-lactide spongy copolymer (PCLA) were tested. At 8 weeks after surgical repair we found that material degradation, expansion, host cell migration, and tissue formation were material related. All the patches were endothelialized on the endocardial surface. Our results indicated that PCLA patches may be the most suitable of the biodegradable biomaterial that we studied for future myocardial cellular engineering.

\section{Methods}

\section{Experimental Animals}

All animal procedures were carried out in compliance with the "Guide to the Care and Use of Experimental Animals" of the Canadian Council on Animal Care and the "Guide for the Care and Use of Laboratory Animals" prepared by the Institute of Laboratory Animal Resources, National Research Council, and published by the National Academy Press, revised 1996. Male syngeneic Lewis rats (Charles River Canada Inc, Quebec, Quebec, Canada) weighing 300 to $350 \mathrm{~g}$ were used for the RVOT replacement procedure.

\section{Biomaterial}

Five materials were used in the in vivo studies: sheets of expanded PTFE,* gelatin sponge (Gelfoam; Pharmacia Co, Kalamazoo,

*Gore-Tex, registered trademark of W. L. Gore \& Associates, Inc, Flagstaff, Ariz.
Mich), PGA (Medifit; JMS Inc, Hiroshima, Japan), and biodegradable poly-L-lactide knitted (KN) or woven (WV) fabric with PCLA (Gunze Ltd, Kyoto, Japan). Rectangular pieces $(7 \times 7 \times 0.4 \mathrm{~mm}$ for PTFE, $7 \times 7 \times 3 \mathrm{~mm}$ for gelatin, $7 \times 7 \times 0.5 \mathrm{~mm}$ for PGA, $7 \times 7 \times 1 \mathrm{~mm}$ for KN-PCLA, $7 \times 7 \times 0.5 \mathrm{~mm}$ for WV-PCLA) were used for the right ventricular wall replacement.

\section{Right Ventricular Outflow Tract Free Wall Resection and Replacement With Patches}

The rats were anesthetized with an intramuscular injection of ketamine hydrochloride $(22 \mathrm{mg} / \mathrm{kg})$, followed by an intraperitoneal injection of sodium pentobarbital $(30 \mathrm{mg} / \mathrm{kg})$. The rats were intubated and ventilated at a rate of 60 cycles/min with a tidal volume of $3 \mathrm{~mL}$ under room air supplemented with oxygen $(2 \mathrm{~L} / \mathrm{min})$ and $1 \%$ to $2.5 \%$ isoflurane.

The rat heart was exposed through a median sternotomy. A purse-string suture (5-6 $\mathrm{mm}$ in diameter) was placed in the free wall of the RVOT. Both ends of the suture were passed through a 22-gauge plastic vascular cannula (Angiocath; Becton Dickinson and Company, Franklin Lakes, NJ), which was used as a tourniquet. The tourniquet was tightened, and the bulging part of the RVOT wall inside the purse-string stitch was lopped off. The tourniquet was briefly loosened to assess whether massive bleeding occurred, indicating that a significant defect had been created in the RVOT. ${ }^{5}$ A patch of nonbiodegradable PTFE or biodegradable gelatin, PGA, KN-PCLA, or WV-PCLA ( $\mathrm{n}=5$ per group) was sutured along the margin of the purse-string suture with an over-and-over method with 7-0 polypropylene (Prolene; Ethicon, Inc, Somerville, NJ) to cover the defect in the RVOT. The tourniquet was released and the purse-string suture was removed. The chest incision was closed in layers with running sutures of 3-0 silk.

Penicillin $\mathrm{G}$ benzathine at $150,000 \mathrm{U} / \mathrm{mL}$ and penicillin $\mathrm{G}$ procaine at $150,000 \mathrm{U} / \mathrm{mL}$ (Penlong XL) was injected intramuscularly $(0.4 \mathrm{~mL}$ per rat). Buprenorphine hydrochloride $(0.01 \mathrm{mg}$ / $\mathrm{kg}$ ) was given subcutaneously every 8 hours for the first 48 hours after the operation. After the operation, rats were monitored in a warm environment until they had completely recovered from the anesthesia and were then returned to their cages.

\section{Morphologic Evaluations of the Patches in Vivo}

Immediately after trimming of the patch to repair the defect in the RVOT, the preoperative patch area was measured with a scale and a digital video camera (DCR-PC110; Sony Corporation, Tokyo, Japan). Eight weeks later the animals were killed with an intraperitoneal injection of $0.2 \mathrm{~mL}$ of pentobarbital sodium (Euthanyl; MTC Pharmaceuticals, Cambridge, Ontario, Canada) after intramuscular injection of 200 units of heparin. Each heart was excised through a median sternotomy and fixed with $10 \%$ phosphatebuffered formalin solution for 2 days. The patch area of the RVOT was remeasured with a scale by using the digital video camera. The ratios of preoperative and postoperative patch areas were calculated and compared among all groups.

The right ventricular free wall was then cut in half through the center of the patch. The thickness of the center of the patch replacing the defect in the RVOT was measured. The ratios of the preoperative and postoperative patch thicknesses were calculated and compared among groups. 


\section{Histologic Studies of the Patches in Vivo}

The 8-week-old implanted patches were processed with JFC solution (Milestone Srl, Sorisole, Italy), embedded in paraffin, and sectioned to yield $3-\mu \mathrm{m}$ thick specimens. The sections were stained with hematoxylin and eosin as described in the manufacture's specifications (Sigma Diagnostics, St Louis, Mo), with Masson trichrome to assess the extracellular matrix, ${ }^{6,7}$ with a monoclonal antibody against vimentin (1:2000, monoclonal V9; Sigma) to assess fibroblast growth, and with an antibody against factor VIII (Polyclonal 1:2000; DAKO Corporation, Carpinteria, Calif) to identify endothelial cells.

\section{Histologic Analysis of the Implanted Patches}

Five different 400-power microscopic fields (ECLIPSE-TE200; Nikon Corporation, Tokyo, Japan) of each section of the RVOT were randomly selected and photographed with a digital camera (Coolpix; Nikon). All the digital images of morphologic and histologic characteristics of the patch portion of the RVOT were analyzed with the National Institutes of Health Image program (version 1.62; National Institutes of Health, Springfield, Va). Briefly, after tracing of the outline of the target areas in the section and subtraction of the backgrounds, the color images were converted to corresponding gray images. A fixed threshold (90 in 255 grades) of gray color scale determined automatically the number of nuclei staining positively for hematoxylin.

\section{Protein Assay of Scaffolds}

The protein content in the remaining half of the patch was extracted. After being washed with buffer, the patch was homogenized with protein extraction solution $(50-\mathrm{mmol} / \mathrm{L}$ tris[hydroxymethyl aminomethane hydrochloride, $0.5-\mu \mathrm{g} / \mathrm{mL}$ aprotinin, $0.5-$ $\mu \mathrm{g} / \mathrm{mL}$ pepstatin $\mathrm{A}$, and $0.5-\mu \mathrm{g} / \mathrm{mL}$ leupeptin) and centrifuged at $4^{\circ} \mathrm{C}$ at $14,000 \mathrm{rpm}$ for 15 minutes. The supernatant was collected. Serial bovine serum albumin solutions were prepared for the standard protein solution as follows: $2,1,0.5,0.25,0.125$, and $0.0625 \mathrm{mg} / \mathrm{mL}$ with $50-\mathrm{mmol} / \mathrm{L}$ tris(hydroxymethyl)aminomethane hydrochloride ( $\mathrm{pH} 7.4$ ). The $495 \mu \mathrm{L}$ of protein assay solution (BIO-RAD Dye and 0.9\% sodium chloride at 5:1; Bio-Rad Laboratories Inc, Hercules, Calif) and $5 \mu \mathrm{L}$ of each protein standard or sample solution were mixed and read with a spectrophotometer (model DU 640; Beckman Coulter, Inc, Fullerton, Calif) at a wavelength of $595 \mathrm{~nm}$. A standard curve was constructed to measure protein concentrations. Gelatin patches were excluded in the assay, because gelatin is also measured by the protein assay. All other patch materials had no measurable protein content. The patch protein content per cubic millimeter was obtained by dividing the protein content by the patch cubic volume.

\section{Data Analysis}

All data are expressed as mean \pm SE. StatView statistical software (SAS Institute Inc, Cary, NC) was used to analyze statistical data. The changes between the preoperative and postoperative patch areas and thicknesses in each group were compared by a paired $t$ test. Comparisons among the five groups were performed by 1-way analysis of variance. The Scheffé test was used to specify differences among groups when the $\mathrm{F}$ ratio of the analysis of variance was significant.
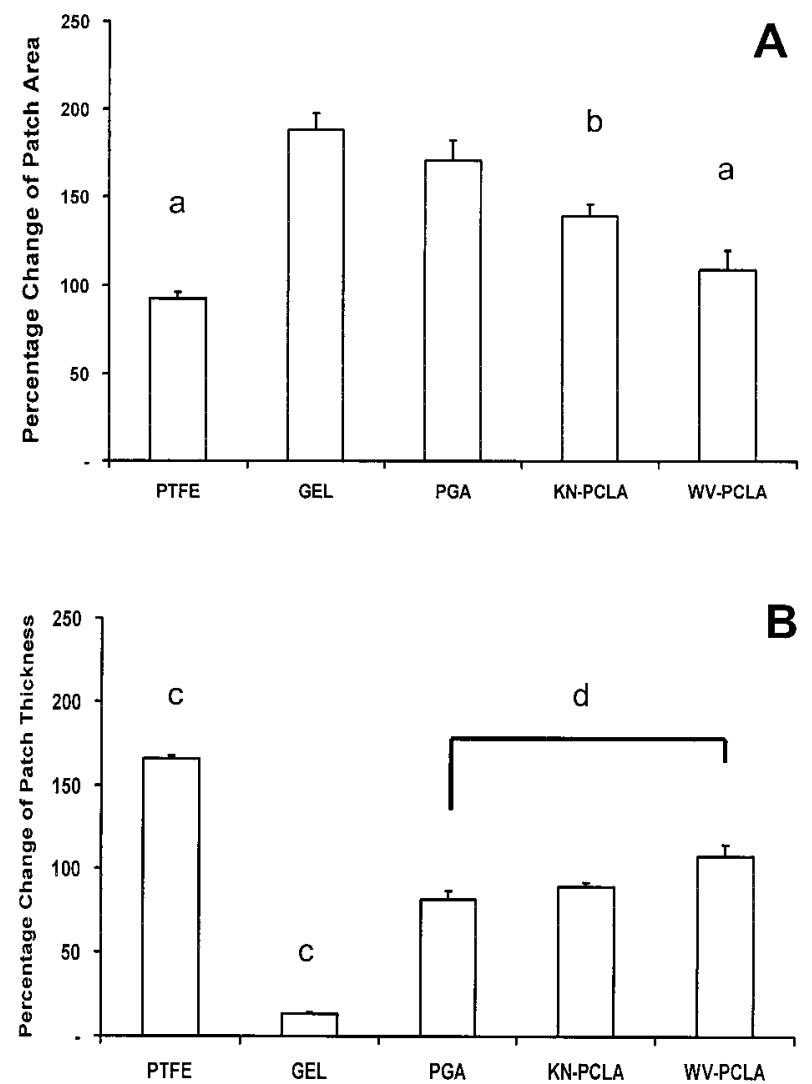

Figure 1. Percentage changes of patch area (A) and thickness (B) after 8 weeks of implantation into defect in RVOT of adult rats. Nonbiodegradable PTFE graft and four biodegradable grafts (gelatin [GEL], PGA, KN-PCLA, and WV-PCLA) were tested. Only PTFE and WV-PCLA grafts did not change in area. Other grafts increased in area. Although PTFE patch itself did not change, its thickness increased $165.3 \%$ because of fibroblast growth and collagen deposition on both sides of patch. Gelatin patch thinned to $13 \%$ of its preoperative thickness and was thinner $(P<.01$, $P<.01, P<.01$, and $P<.01$, respectively) than PTFE, PGA, KN-PCLA, and WV-PCLA patches. In the PGA and KN-PCLA patches but not in the WV-PCLA patch, thickness decreased $(P<$ $.05)$ during 8 weeks in RVOT. $a, P<.01$ compared with GEL and PCA; $b, P<.05$ compared with GEL; $c, P<.01$ compared with PGA and PCLA; $d, P<.05$ PGA compared with WV-PCLA.

\section{Results}

Biomaterial Area and Thickness Changes in Vivo

There were no significant differences in preoperative patch area among the five biomaterials (PTFE $16.6 \pm 1.5 \mathrm{~mm}^{2}$, gelatin $16.8 \pm 1.5 \mathrm{~mm}^{2}$, PGA $17.3 \pm 1.2 \mathrm{~mm}^{2}$, KN-PCLA $16.9 \pm 1.0 \mathrm{~mm}^{2}$, WV-PCLA $17.3 \pm 2.1 \mathrm{~mm}^{2}, \mathrm{n}=5$ for each group).

Patch area. Eight weeks after implantation, the changes in the patch area (the ratios of postoperative to preoperative values) were different among the five materials (Figure 1, $A$ ). The nonbiodegradable PTFE patch did not change in 


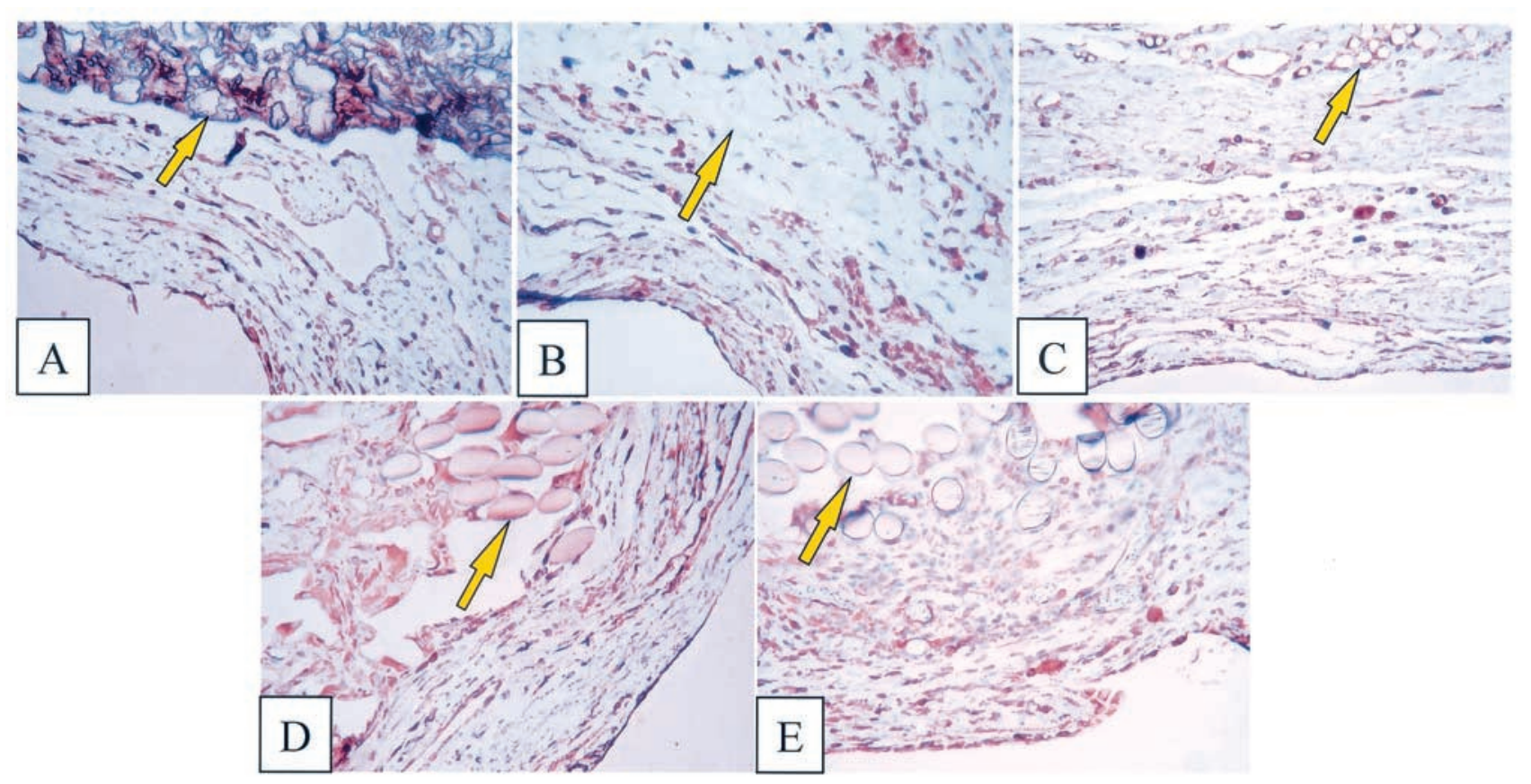

\begin{abstract}
Figure 2. Host cell growth in patch scaffolds at 8 weeks after implantation into defect in RVOT of adult rats (original magnification $\times 200$ ). Patches were stained for fibroblasts with monoclonal antibody against vimentin. Patches studied were PTFE (A), gelatin (B), PGA (C), KN-PCLA (D), and WV-PCLA (E). Host fibroblasts (brownish cells) grew onto surface of PTFE scaffold (yellow arrow) and grew into gelatin, PGA, KN-PCLA, and WV-PCLA scaffolds (yellow arrows).
\end{abstract}

area. However, the biodegradable gelatin and PGA patches expanded significantly $(P<.01$ for both) relative to the PTFE patch area. The KN-PCLA patch was larger $(P<.05)$ than the PTFE patch but significantly smaller $(P<.05)$ than the gelatin patch. The KN-PCLA and PGA patch areas did not differ. The WV-PCLA patch did not expend in area and was not statistically different in size from that of the $\mathrm{KN}$ PCLA patch. The KN-PCLA patch size was smaller in area than the gelatin and PGA patches $(P<.01$ for both).

Patch thickness. The thickness of the biomaterials changed during the 8 weeks of implantation (Figure 1,B). Although the PTFE patch itself did not change, its thickness increased $165.3 \%$ because of fibroblast growth and collagen deposition on both sides of the patch. The gelatin patch thinned to $13 \%$ of its preoperative thickness and was thinner $(P<.01$ in all cases) than the PTFE, PGA, KN-PCLA, and WV-PCLA patches. The PGA and KN-PCLA patches, but not the WV-PCLA patch, decreased in thickness $(P<.05$, $P<.05)$ during the 8 weeks in the RVOT.

\section{Cell Migration and Extracellular Matrix Formation in the Biomaterials}

Only the surfaces of the PTFE patch were coated with fibroblasts (Figure 2, A) and collagen (Figure 3, A). No cellular infiltration was evident within the PTFE patch. The
PTFE patch remained unchanged. In contrast, fibroblast ingrowth (Figure 2, $B-E$ ) and collagen formation (Figure 3, $B-E$ ) were present throughout the gelatin, PGA, KN-PCLA, and WV-PCLA patches. Of the biodegradable materials, the highest cell number per unit area was measured in the KN-PCLA patch (Figure 4). No significant differences in cell numbers were found among the gelatin, PGA, and WV-PCLA patches. Two of five gelatin patches had foci of inflammatory cells. The gelatin, PGA, KN-PCLA, and WVPCLA materials were partially absorbed and replaced by fibroblasts and collagen (Figure 2, $B-E$, and Figure 3, $B-E$ ).

At 8 weeks after patch implantation, the patch protein levels (measurement of both the cellular and matrix protein levels) of $7.8 \pm 1.4 \mu \mathrm{g} / \mathrm{mm}^{3}$ in the KN-PCLA patches $(\mathrm{n}=5)$ and $7.4 \pm 1.6 \mu \mathrm{g} / \mathrm{mm}^{3}$ in the WV-PCLA patches $(\mathrm{n}=4)$ were greater $(P<.01$ and $P<.05$, respectively) than the protein levels of $1.8 \pm 0.5 \mu \mathrm{g} / \mathrm{mm}^{3}$ in the PGA patch $(\mathrm{n}=5)$ and 1.0 $\pm 0.3 \mu \mathrm{g} / \mathrm{mm}^{3}$ in the PTFE patch $(\mathrm{n}=4)$. Because the gelatin patch was itself proteinaceous, its protein levels were not measured.

\section{Endothelialization}

At 8 weeks after implantation all the patches were endothelialized on their endocardial surfaces (Figure 5). No thrombi were observed on the patches. 


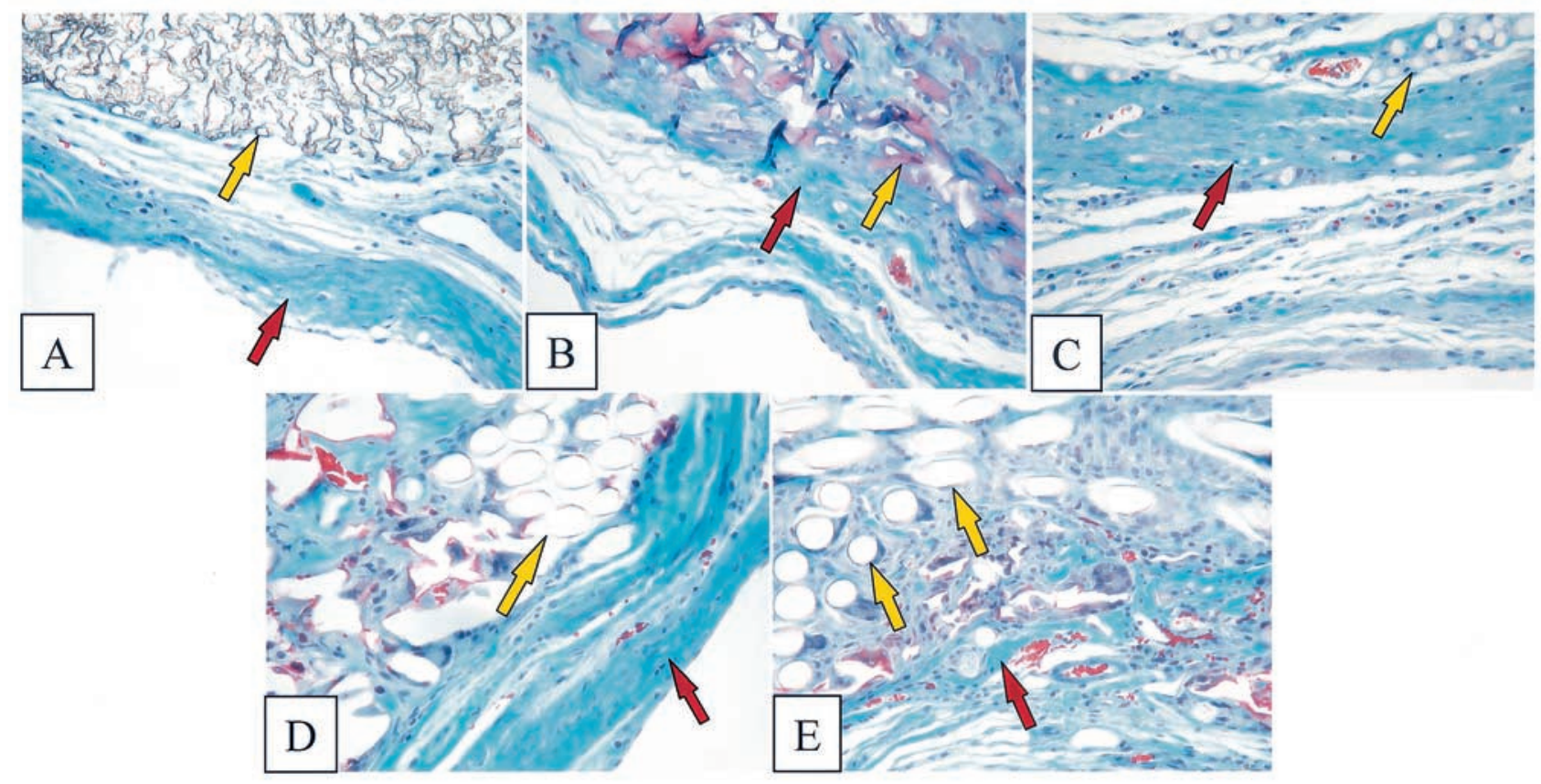

Figure 3. Extracellular matrix in patch scaffolds at 8 weeks after implantation into defect in RVOT of adult rats (original magnification $\times 200$ ). Patches studied were PTFE (A), gelatin (B), PGA (C), KN-PCLA (D), and WV-PCLA (E). Collagen in patches was stained with Masson trichrome. Collagen (light green in color, red arrows) was found in all biomaterials, as indicated by yellow arrows.

\section{Discussion}

Current bioprosthetic replacement devices expose the patient to the risks of thrombosis, the problem of limited durability, and frequently the requirement for reoperation because of the lack of patch growth. ${ }^{8}$ The important role for the scaffolds in building biocompatible and viable prostheses to repair heart defects is to facilitate host cell attachment and proliferation and to enhance the formation of the extracellular matrix. It is well known in cell culture studies that unless cultured cells can attach to the scaffolding, they will die. ${ }^{9}$ Cellular growth is required in the scaffold to form tissue and extracellular matrix. Seeding different cell types in vitro can affect the composition of the extracellular matrix. Smooth muscle cells will form collagen and elastin, whereas fibroblasts will usually synthesize only collagen.

A biodegradable scaffold should be preferable to a nonbiodegradable scaffold. A biodegradable scaffold can be replaced by the patient's own cells and will be treated as a foreign body for only a limited time. The PTFE patch has been accepted worldwide as one of the most reliable nonbiodegradable synthetic materials ${ }^{10}$ in terms of durability, low thrombogenicity, and comfortable handling for surgery. Unfortunately, it does not grow as a child's heart or blood vessel grows, and it carries increased susceptibility to infection. ${ }^{11}$ In this study PTFE was used as a control patch for comparison purposes with the biodegradable patches that

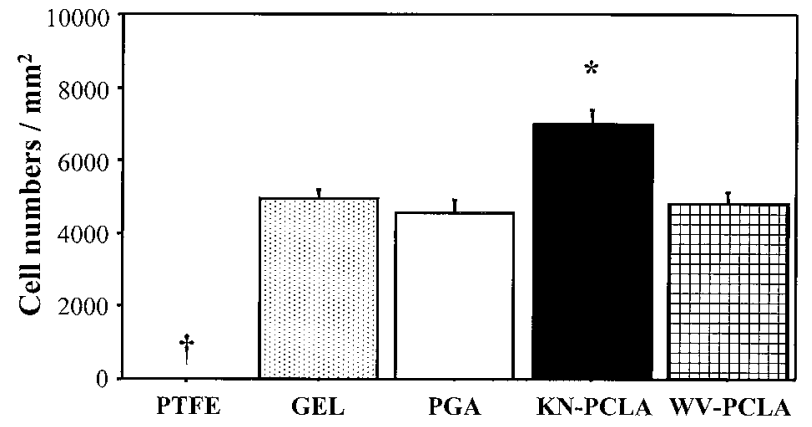

Figure 4. Numbers of host cells in patch scaffolds at 8 weeks after implantation into defect in RVOT of adult rats. Patches studied (n = 5 for each biomaterial) were PTFE, gelatin (GEL), PGA, KN-PCLA, and WV-PCLA. KN-PCLA patch contained largest number of cells. PTFE patch contained no cells. Asterisk indicates $\boldsymbol{P}<.01$; dagger indicates $\boldsymbol{P}<.001$ versus other groups.

we studied. The biodegradable gelatin sponge (Gelfoam) was tested because of our earlier works. ${ }^{5,12,13}$ The use of fetal cardiomyocytes the pliable and fragile gelatin scaffold enabled us to create in vitro and in vivo beating patches. The biodegradable nonwoven PGA mesh was studied because it is one of the most common and attractive synthetic biodegradable polymers for tissue engineering field. ${ }^{14}$ However, this PGA has recently been used in combination with an- 


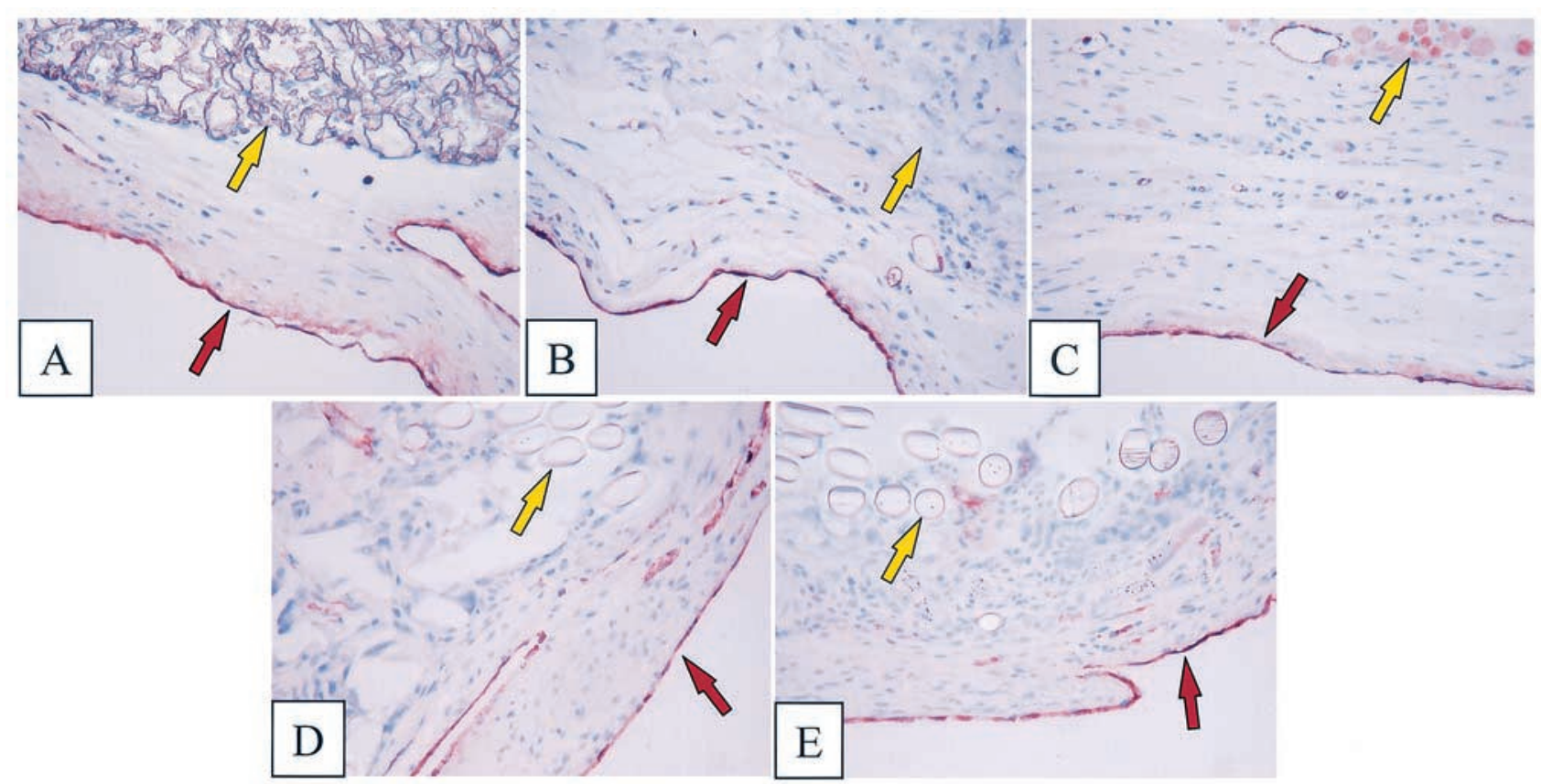

Figure 5. Endothelialization of patch scaffolds at 8 weeks after implantation into defect in RVOT of adult rats (original magnification $\times 200$ ). Endothelial cells were identified by immunohistochemical staining for factor VIII. Patches studied were PTFE (A), gelatin, (B), PGA (C), KN-PCLA (D), and WV-PCLA (E). Endothelial cells (brownish cells, red arrows) covered endocardial surfaces of all biomaterial scaffolds (yellow-arrows).

other biodegradable polymer, $7,15,16$ because nonwoven meshes of PGA fibers lack structural stability and often cannot maintain their original structure during tissue development. ${ }^{17}$ Of special interest to us was the PCLA patch with a biodegradable spongy matrix, reinforced on the outside with a knitted or woven poly-L-lactide fabric that takes between 1 and 2 years to biodegrade (information provided by the manufacturer). We thought that the spongy matrix would favor in vitro and in vivo cell growth and might be suitable for in vitro autologous seeding of smooth muscle cells or stem cell-derived cardiomyocytes. Also, the outer fabric of PCLA patch should provide structural stability and permit sufficient time for in vivo maturation of the seeded cells in the contracting ventricular wall. Kim and Mooney ${ }^{17}$ demonstrated that the poly-L-lactide-bonded PGA matrices had a high cellularity and maintained their predefined structure more than did the unbonded PGA matrices during the process of tissue development in vitro. Their report suggests that the combined scaffold structure, like the PCLA patch in our study, should allow the maintenance of the configuration and dimension of the original matrices and the development of a new tissue in a predefined 3-dimensional structure.

Because Carver, ${ }^{18}$ Freed, ${ }^{19}$ and their colleagues have shown that mechanical stimuli and pressure can enhance cellular formation of extracellular matrix and tissue regen- eration, we used an animal model that simulates the in vivo conditions to which a tissue patch would be exposed clinically. In addition to the mechanical forces of myocardial contractility, the patch is influenced by the myocardial tissue milieu and is in direct contact with the blood in the right ventricular chamber. An 8-week period of in vivo testing was arbitrarily chosen as being sufficiently long to detect graft failure and to compare the various scaffolds.

The PTFE scaffold did not change in its dimensions or break down after 8 weeks as a patch in the RVOT. Compared with the biodegradable patches, PTFE permitted only surface cell growth that consisted predominantly of fibroblasts. Fibrous tissue was firmly attached onto both surfaces of the patch. No inflammatory infiltration was noted. The gelatin patch was unsuitable as a patch because of its marked expansion and thinning. The gelatin scaffold was almost completely degraded and infiltrated with fibroblasts. Additionally, some inflammatory foci were found. Because this phenomenon was found in one of our earlier studies, ${ }^{5}$ we believe that the inflammatory reaction was caused by a xenogenic reaction to porcine skin proteins in the sponge. The nonwoven PGA patch increased in surface area and thinned after 8 weeks in the RVOT. The scaffold itself was almost completely degraded and infiltrated with fibroblasts. The expansion of the patch size during the 8 weeks indicated either that the scaffold biodegradation process was not 
in synchrony with the fibroblast colonization and collagen deposition or that fibroblast colonization and collagen deposition were insufficient to prevent the changes in patch surface area and thickness.

Similar results to the PGA patch were observed with the knitted PCLA patch except that fibroblast colonization per unit area was greater and more localized in the knitted matrix than in the PGA patch. Also, collagen formation appeared to be greatest in the KN-PCLA patch. The dimension of the WV-PCLA patch was almost unchanged during the 8 weeks, despite the spongy matrix scaffold degradation, fibroblast infiltration, and collagen deposition in the sponge. In contrast, the KN-PCLA patch permitted more host fibroblast infiltration than did the WV-PCLA patch. This finding is consistent with previous reports that studied woven and knitted Dacron polyester fabric grafts. ${ }^{20,21}$

The endocardial surface was covered by endothelial cells in all the biomaterials tested. As Scott and colleagues ${ }^{22}$ demonstrated, our findings indicate that host endocardial endothelial cells or endothelial progenitor cells were involved in endothelialization of the endocardium. The necessity of seeding endothelial cells in biomaterials remains controversial. To avoid thrombosis, some investigators have constructed conduits seeded with endothelial cells before implantation. ${ }^{23}$ On the other hand, Shi and coworkers ${ }^{24}$ demonstrated that the rapidity with which the specimens are fixed affects the identification of endothelium in explanted synthetic aortic grafts obtained from the patients. They also suggested that endothelialization of synthetic arterial grafts might occur more frequently in patients than previously recognized.

If a biodegradable scaffold is infiltrated primarily with fibroblasts that form a fibrous structure, localized dilatation of the patch may occur similarly to a ventricular aneurysm formed after a transmural myocardial infarction. However, Malm and colleagues ${ }^{25}$ reported that unseeded absorbable, nonwoven patches made from polyhydroxybutyrate implanted as transannular patches into the RVOT in 13 weanling sheep could be used as a scaffold for tissue regeneration in low-pressure systems. Our histologic results demonstrated that the patch cells and extracellular matrix in the biodegradable scaffolds were predominantly fibroblasts and collagen. It has been unclear whether the nonmuscular regenerated tissue can tolerate right ventricular pressure without dilatation during the long term unless a strong and durable biodegradable material is used. Even after the RVOT reconstruction with a transannular patch or conduit, the implanted material may be exposed to residual stenosis because of remaining thick trabecular muscle through the RVOT to pulmonary artery or may be exposed to pulmonary hypertension. In these cases, the biomaterial would require more strength to prevent its dilatation because of increased right ventricular pressure overload.
To avoid host overcolonization of the scaffold with fibroblasts and eventual scar formation, in vitro autologous cell seeding may be essential in cardiac tissue engineering. In vitro seeding of a biodegradable material with cultured smooth muscle cells would permit formation of a muscular tissue within the scaffold and the formation of elastin extracellular matrix in addition to collagen formation ${ }^{7,26}$ Shinoka and associates ${ }^{23}$ have demonstrated the benefits of in vitro autologous cell seeding to form mature autografts from synthetic biodegradable tubular scaffolds. We have also found morphologic differences between the cell-seeded and nonseeded gelatin patches. ${ }^{5}$ The elastic properties of elastin and strength of collagen formed by the implanted smooth muscle cells and the capacity of smooth muscle cells to proliferate and hypertrophy in response to increased pressure should prevent patch dilatation and thinning.

One limitation of this study is the small size of the patches in the right ventricular outflow tract. Our ultimate goal is to create a beating conduit that will provide pulsatile perfusion to the lungs. Our preliminary study ${ }^{12}$ with seeding a gelatin patch with fetal cardiomyocytes suggests that a beating patch may be possible in the right ventricular outflow tract. However, allogeneic cells are eventually rejected, and a better biomaterial would be required before assessing cell-seeded grafts. Therefore this study reviewed alternative scaffolds that can be used for cell seeding. It should be possible to seed a biodegradable scaffold with beating cardiomyocytes derived from mesenchymal stem cells and to use the scaffold in vivo to induce pulsatile pulmonary perfusion. A challenge for all biodegradable patches is to seed the correct number and proportion of myocytes, endothelial cells and fibroblasts so that the graft can develop in vivo into a structurally strong tissue that does not dilate in response to intraventricular pressures. With a large graft, selective endothelial cell seeding of the endocardial surface may be necessary before implantation to ensure complete in vivo endothelial coverage of the graft's endocardial surface.

In summary, the unique structure of the PCLA patch with a spongy matrix favors in vivo cell colonization relative to the other patches tested. Its outer poly-L-lactide fabric layer also offers advantages relative to the other biodegradable materials we studied.

We thank Yoshito Ikada, MD, PhD, and Shin'ichiro Morita, MAgr, for providing PCLA material. We thank Tetsuro Sakai, $\mathrm{MD}, \mathrm{PhD}$, for his technical advice.

\section{References}

1. Danielson GK, Anderson BJ, Schleck CD, Ilstrup DM. Late results of pulmonary ventricle to pulmonary artery conduits. Semin Thorac Cardiovasc Surg. 1995;7:162-7.

2. Stark J, Bull C, Stajevic M, Jothi M, Elliott M, de Leval M. Fate of subpulmonary homograft conduits: determinants of late homograft failure. J Thorac Cardiovasc Surg. 1998;115:506-16.

3. Homann M, Haehnel JC, Mendler N, Paek SU, Holper K, Meisner H, et al. Reconstruction of the RVOT with valved biological conduits: 25 
years experience with allografts and xenografts. Eur J Cardiothorac Surg. 2000;17:624-30.

4. Oechslin EN, Harrison DA, Harris L, Downar E, Webb GD, Siu SS, et al. Reoperation in adults with repair of tetralogy of Fallot: indications and outcomes. J Thorac Cardiovasc Surg. 1999;118:245-51.

5. Sakai T, Li RK, Weisel RD, Mickle DA, Kim ET, Jia ZQ, et al. The fate of a tissue-engineered cardiac graft in the right ventricular outflow tract of the rat. J Thorac Cardiovasc Surg. 2001;121:932-42.

6. Young B, Burkitt HG, Heath JW, Lowe JS, Stevens A, Wheater PR. Supporting/connective tissues: In: Young B, Heath JW, editors. Wheater's functional histology: a text and colour atlas. 4th ed. New York: Churchill Livingstone; 2000. p. 65-79.

7. Watanabe M, Shin'oka T, Tohyama S, Hibino N, Konuma T, Matsumura $\mathrm{G}$, et al. Tissue-engineered vascular autograft: inferior vena cava replacement in a dog model. Tissue Eng. 2001;7:429-39.

8. Mayer JE Jr, Shin'oka T, Shum-Tim D. Tissue engineering of cardiovascular structures. Curr Opin Cardiol. 1997;12:528-32.

9. Kang SW, Lim SW, Choi SH, Shin KH, Chun BG, Park IS, et al. Antisense oligonucleotide of cluster in mRNA induces apoptotic cell death and prevents adhesion of rat ASC-17D Sertoli cells. Mol Cells. 2000;10:193-8.

10. Izutani H, Gundry SR, Vricella LA, Xu H, Bailey LL. Right ventricular outflow tract reconstruction using a GoreTex membrane monocusp valve in infant animals. ASAIO J. 2000;46:553-5.

11. Uemura H, Yagihara T, Kawahira Y, Yoshikawa Y, Kitamura S. Total cavopulmonary connection in children with body weight less than 10 kg. Eur J Cardiothorac Surg. 2000;17:543-9.

12. Li RK, Jia ZQ, Weisel RD, Mickle DA, Choi A, Yau TM. Survival and function of bioengineered cardiac grafts. Circulation. 1999;100(19 Suppl):II-63-9.

13. Li RK, Yau TM, Weisel RD, Mickle DA, Sakai T, Choi A, et al. Construction of a bioengineered cardiac graft. J Thorac Cardiovasc Surg. 2000;119:368-75.

14. Shinoka T, Breuer CK, Tanel RE, Zund G, Miura T, Ma PX, et al. Tissue engineering heart valves: valve leaflet replacement study in a lamb model. Ann Thorac Surg. 1995;60(6 Suppl):S513-6.
15. Stock UA, Nagashima M, Khalil PN, Nollert GD, Herden T, Sperling JS, et al. Tissue-engineered valved conduits in the pulmonary circulation. J Thorac Cardiovasc Surg. 2000;119:732-40.

16. Hoerstrup SP, Sodian R, Daebritz S, Wang J, Bacha EA, Martin DP, et al. Functional living trileaflet heart valves grown in vitro. Circulation. 2000;102(19 Suppl 3):III-44-9.

17. Kim BS, Mooney DJ. Engineering smooth muscle tissue with a predefined structure. J Biomed Mater Res. 1998;41:322-32.

18. Carver SE, Heath CA. Influence of intermittent pressure, fluid flow, and mixing on the regenerative properties of articular chondrocytes. Biotechnol Bioeng. 1999;65:274-81.

19. Freed LE, Langer R, Martin I, Pellis NR, Vunjak-Novakovic G. Tissue engineering of cartilage in space. Proc Natl Acad Sci U S A. 1997; 94:13885-90.

20. Haverich A, Oelert H, Maatz W, Borst HG. Histopathological evaluation of woven and knitted Dacron grafts for right ventricular conduits: a comparative experimental study. Ann Thorac Surg. 1984;37: 404-11.

21. Yu TJ, Ho DM, Chu CC. Bicomponent vascular grafts consisting of synthetic absorbable fibers. Part II: in vivo healing response. J Invest Surg. 1994;7:195-211.

22. Scott SM, Barth MG, Gaddy LR, Ahl ET Jr. The role of circulating cells in the healing of vascular prostheses. $J$ Vasc Surg. 1994;19:585-93.

23. Shinoka T, Shum-Tim D, Ma PX, Tanel RE, Isogai N, Langer R, et al. Creation of viable pulmonary artery autografts through tissue engineering. J Thorac Cardiovasc Surg. 1998;115:536-46.

24. Shi Q, Wu MH, Onuki Y, Ghali R, Hunter GC, Johansen KH, et al. Endothelium on the flow surface of human aortic Dacron vascular grafts. J Vasc Surg. 1997;25:736-42.

25. Malm T, Bowald S, Bylock A, Busch C, Saldeen T. Enlargement of the right ventricular outflow tract and the pulmonary artery with a new biodegradable patch in transannular position. Eur Surg Res. 1994;26: 298-308.

26. Shinoka T, Ma PX, Shum-Tim D, Breuer CK, Cusick RA, Zund G, et al. Tissue-engineered heart valves: autologous valve leaflet replacement study in a lamb model. Circulation. 1996;94(9 Suppl): II-164-8. 